\title{
Cooperative Selection and Communications for Internet of Everything in 6G Ultra-mMTC loT Networks
}

This paper was downloaded from TechRxiv (https://www.techrxiv.org).

\section{LICENSE}

CC BY 4.0

SUBMISSION DATE / POSTED DATE

29-09-2021 / 30-09-2021

CITATION

Huang, He; Hu, Su (2021): Cooperative Selection and Communications for Internet of Everything in 6G UltramMTC loT Networks. TechRxiv. Preprint. https://doi.org/10.36227/techrxiv.16699597.v1

$\mathrm{DOI}$

10.36227/techrxiv.16699597.v1 


\title{
Cooperative Selection and Communications for Internet of Everything in 6G Ultra-mMTC IoT Networks
}

\author{
H. Huang, Member, IEEE, S. Hu*, Member, IEEE
}

\begin{abstract}
Although Cooperative Communications (CC) already exists in 4G LTE/5G, performance improvement is just not enough, hence, how to apply collaborative technology in $6 \mathrm{G}$ to achieve Internet of Everything and improve communication quality significantly is one of most important issues. As 5G communications standard has gradually established recently, CC has been one of most critical communication technologies which plays a founding role on Internet of Everything in 6G networks. Thus, in this study we propose new 6G enabled ultra-massive Machine Type Communication (mMTC) framework based on space-ground integrated networks, and consider that collaborative ideology has been regarded as foundation theory which widely exists in future multiple hybrid scenarios, such as, CognitiveInternet of Things (C-IoT) networks, UAVs (Unmanned Aerial Vehicles) communications, air-space-ground integrated networks, underwater acoustic communication and so on. Besides, we discuss other key communication technologies that are closely combined with unique communication scenes, and elaborate critical problems that shall be solved in 6G ultra-mMTC Internet of Everything networks. Furthermore, we discuss extreme lowcomplexity and fast ultra-massive relays selection algorithm to apply in all sorts of future 6G scenarios. At last, it is concluded that for arbitrary two communication points (source/destination devices, sensors, relays, IoT nodes and so on), ideology of nodes selection of collaborative communication is theory of foundation to realize and optimize communication transmission in 6G Internet of Everything ultra-mMTC networks.
\end{abstract}

Index Terms - 6G ubiquitous networks, Internet of Everything, collaborative ideology, ultra-mMTC

\section{INTRODUCTION}

With rapid development of wireless communications, 5G has been gradually applied in all aspects of work, study and life, in the light of further improving communication transmission quality substantially, recently research plans of 6G are put forward to develop related new core communications techniques, such as Artificial Intelligence (AI), mm-wave communications, Full-Duplex (FD), Free-Space Optical (FSO), etc $[1,2]$. Cooperative Communications (CC) has been

Corresponding author: husu@uestc.edu.cn

H.Huang and S.Hu are with the National Key Laboratory on Communications, University of Electronic Science and Technology of China, Chengdu 611731, China, e-mail: (huanghe@uestc.edu.cn, husu@uestc.edu.cn) This work was supported by National Natural Science Foundation of China (Grant No. XXXXXXXX), China Postdoctoral Science Foundation (Grant No. regarded as pivotal technology to make arbitrary two communication points (such as, mobile source/destination devices, sensors, relays, mMTC Internet of Things nodes and so on) establish connection, and in future Internet of Everything networks device-to-device communication will be enabled and optimized with the aid of massive/ultra-massive machine Internet of Things (IoT), therefore it is very important to consider future essential communication technologies based on collaborative selection and interconnection, on this basis, we can make further efforts to obtain optimal transmission Quality of Service (QoS) in different cooperative topology networks [3, 4].

Multiple nodes collaborative communications will be extensively existed in all kinds of B5G/6G networks, for example, Cognitive-IoT networks, UAVs communications, space-ground integration networks, underwater acoustic communications, FSO communications, smart city, vehicular networks, cloud-fog-edge intelligent computing and so on. In these scenarios, cooperative transmission plays an auxiliary role for other 6G communication technologies to establish inter-communication between sources and destinations, and optimize device-to-device transmission performance [5-7]. On the other hand, in order to comprehensively improve QoS more effectively, critical communication technologies are closely linked with cooperative topology networks, therefore, multiple relays with massive MIMO (Multi-Input Multi-Output), largescale sensors with multiple access, space-air-ground integrated network with relays of air and space, ultra dense networks with ultra-massive IoT nodes, full-duplex cooperative transmission are comprehensive issues that shall be investigated in future 6G Internet of Everything ultra-mMTC hybrid and ubiquitous networks $[1,5,7]$.

Moreover, effectiveness of collaborative core algorithms are fundamental research highlights-how to construct advantage topology network structure and obtain more efficient transmission quality. At present, cooperative selection algorithms mainly include single node selection and multiple nodes selection, the former contains best node selection, $\mathrm{N}$-th

XXXXXXXX), National Natural Science Foundations of China under (No.61971092), Sichuan Science and Technology Program (No.2020JDJQ0023), partially supported by Fundamental Research Funds for the Central Universities (No.ZYGX2019J123) 
best node selection, partial node selection, random single node selection and so on $[9,10]$, the latter contains several classical and available nodes subsets selection. Generally speaking, algorithms based on subsets selection are relatively more effective than algorithms based on single selection [9-15].

Stated thus, in this study, we make contributions as follows,

1) We propose novel Internet of Everything ultra-mMTC network topology structure-6G hybrid and ubiquitous intelligent transmission cooperative system, and implement interconnection of arbitrary two communication nodes in airspace-ground networks. In addition, we reveal essential characteristics of Internet of Everything networks-existence of optimal path and optimum transmission for device-to-device communication.

2) We profoundly discuss key techniques with cooperative transmission in different 6G communication scenarios, highlight foundation role of cooperative theory in future integrated networks and analyze how these techniques collectively improve end-to-end QoS through efficient collaborate schemes.

3) After summing up crucial problems that shall be solved in 6G hybrid and ubiquitous networks, we also analyze existed cooperative core algorithms and make some comparison in several aspects include performance, applicable scenarios and advantages and disadvantages. Moreover, it is concluded that cooperative selection and communications lays the foundations of Internet of Everything, and collaborative optimal communications is one of most important research hotspots to build future 6G dynamic networks.

4) Completely different from [9-15], this study originally proposes fast ultra-massive core relays selection algorithm, proposed scheme not only has exceedingly lower computational complexity than other cooperative algorithms, but also can be used for finding out multiple optimal relays subsets selection solutions according to different features of intelligent relays in all types of $6 \mathrm{G}$ specific scenarios.

\section{PROPOSED 6G ULTRA-MMTC INTELLIGENT TRANSMISSION COOPERATIVE SYSTEM IN INTERNET OF EVERYTHING NETWORKS}

In this part, as shown in Fig.1, firstly we set up 6G hybrid and ubiquitous ultra-mMTC cooperative networks based on spaceground communications, and point out the key of achieving communication and further optimizing transmission quality is to realize different cooperative networks interconnection efficiently in multiple related scenarios. Consequently, in the era of 6G intelligent communication, when ultra-massive IoT devices perform various intelligent functions of receive, selection, compute and forward, for one thing, we can expand communication coverage widely through building or connecting local collaborative networks, for another, it is necessary to select appropriate relays/sensors/IoT nodes in different networks, so that wider ubiquitous networks will be established effectively to ensure high quality of communication transmission. On the whole, better selection is essential in ultramassive IoT networks for $6 \mathrm{G}$ Internet of Everything.

Secondly, in Fig.1 we present 6G ubiquitous networks with several fundamental cooperative networks, for example, it shows that if one of arbitrary mobile users A in left cellular networks wants to communicate with one of fixed arbitrary user $\mathrm{B}$ in right cellular networks on ground zone, A can connect to B through multiple independent networks at the same time. In addition, in this process by means of advanced artificial intelligence and signals processing techniques, A will firstly select multiple optimized paths and then communicate with B more fast and reliably in newly established heterogeneous networks. In a word, in view of constantly integrating all sorts of independent local collaborative networks, it is challenging to expand communication coverage region and develop corresponding optimal networks structure for a long time to come.

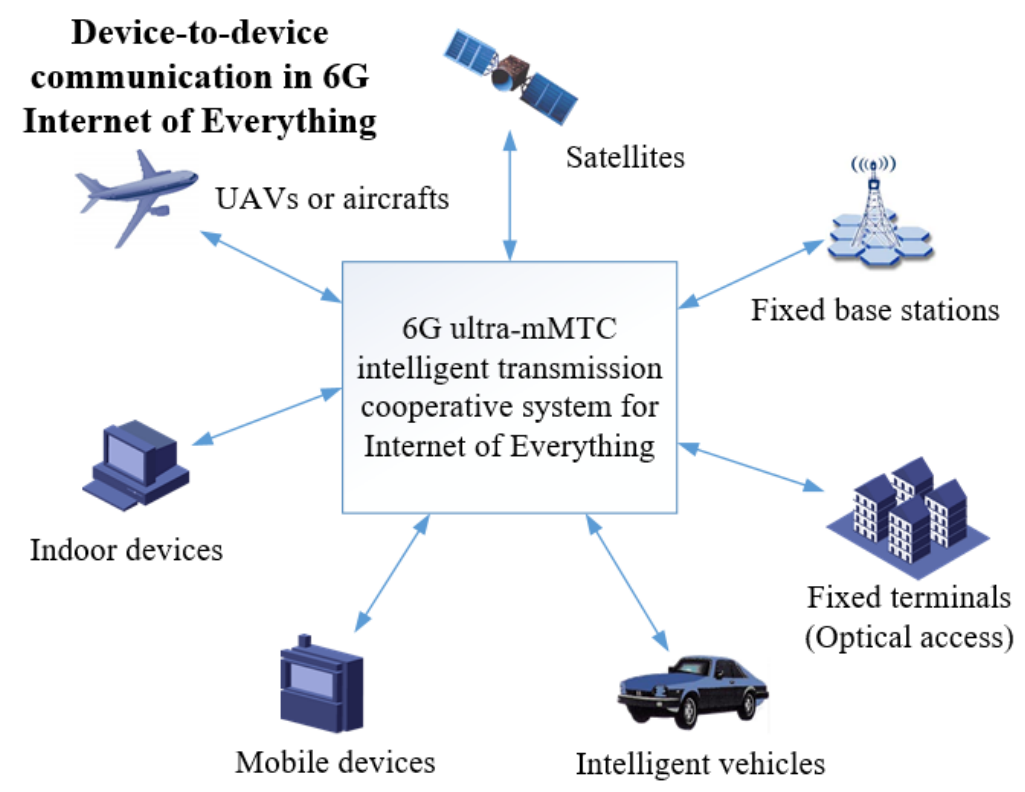

FIGURE2. Internet of everything with 6G intelligent transmission cooperative system in ultra-mMTC cooperative device-to-device networks

On the other hand, according to discussion for selection and communications above, in Fig.2 we propose 6G ultra-mMTC intelligent transmission cooperative system, which contains a large number of intelligent relays and mobile devices in 6G airspace-ground integrated networks such as fixed relays, satellites, laptops, UAVs and so on. It is raised that device-todevice communications (also for fixed relays, satellite, UAVs, mobile devices, etc) for arbitrary two nodes in Fig.2 will be substantially optimized with the aid of proposed scalable cooperative system. Combined with Fig.1 and Fig.2, we observe that arbitrary multiple communication nodes in Fig.1 can achieve highly quality of inter-communication for Internet of Everything in Fig.2, so after creating 6G integrated networks, we set up efficient data-transmission link with the aid of synthetic intelligent transmission and computational algorithms, which takes artificial intelligence as the core technique. Thus, as Fig.2 shows, random sources/destinations/relays/sensors/ IoT nodes or other communication nodes, will be contained in 6G ultra-mMTC intelligent cooperative systems, and wider 
networks will mix more key nodes broadly.

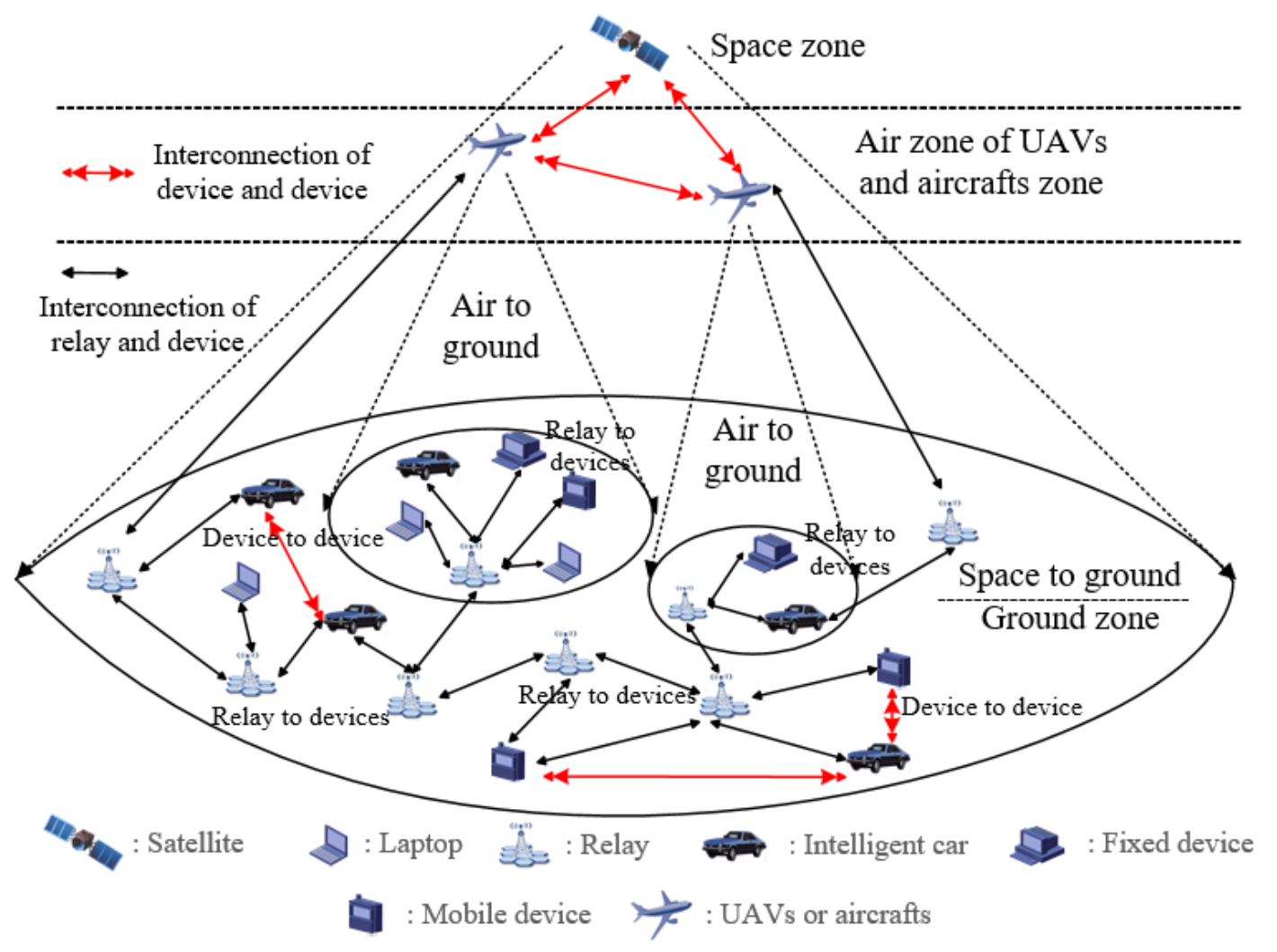

FIGURE1. 6G hybrid and ubiquitous air-space-ground ultra-mMTC networks

\section{COMBINATION OF TECHNIQUES IN 6G ULTRA-MMTC COOPERATIVE NETWORKS}

On the basis of proposed converged 6G ubiquitous networks, in order to markedly improve transmission performance, we summary that development and application of related core technologies are existed in proposed 6G ultra-mMTC hybrid air-space-ground networks. In table 1 , on one hand, we sum up respective cooperative networks features in different communication scenarios to present varied and unique characteristics of collaborative ultra-mMTC transmissions. On the other hand, involved technologies of 6G are widely applied in correlative networks and particular scenarios, such as, Thz communications, artificial intelligence, massive MIMO, Orthogonal Frequency Division Multiple Access (OFDMA), FD communications, device-to-device optimized communications, mm-wave communications, Non-Orthogonal Multiple Access (NOMA), Sparse Code Multiple Access (SCMA), Pattern Division Multiple Access (PDMA), intelligent computation (intelligent cloud-fog-edge computation) and so on. Moreover, several specific techniques listed above are comprehensively related to significantly promote service quality in $6 \mathrm{G}$ hybrid networks.

\section{Problems of CoOperative SELECTIONS AND COMMUNICATIONS IN 6G ULTRA-MMTC NETWORKS}

Along with complex 6G hybrid networks that are discussed above, in order to improve transmission QoS essentially, we summary that there are mainly several problems of major respects for different future ultra-mMTC networks that shall be considered thoroughly, examples are as follows.

*Paths selection and optimization. For arbitrary two nodes in hybrid networks, how to develop multiple optimized or suboptimized selected paths are very important works, main problems include dual-hop path selection and multiple hops paths selection, thus one or more practical paths will firstly be computed, and then be selected to enable efficiency, reliability and security of transmission.

*Single node or nodes subsets selection. In view of abovementioned paths discussion, it points to a deeper problem-nodes selection that which ones should be chosen. In proposed 6G Internet of Everything air-space-ground integrated networks, when various intelligent communication nodes (satellites, unmanned drones, vehicles, mobile devices, IoT nodes/sensors and fixed relays) are considered as collaborative terminals meanwhile, with regard to joint choice of multiple nodes, advanced relays subsets method will lead to appropriate selected nodes to establish multi-path link networks, in addition, how to select better mobile nodes subsets and optimize paths selection synchronously is a more comprehensive problem.

*Resource allocation. Effective resource allocation schemes should be proposed to assign fixed total power to source/destination/CC devices and selected nodes reasonably, on this basis, achieving more efficient allocation schemes for changeable relays and analyze correlative performance optimization in the process of nodes mobility for different communication systems. 
*NP-hard (Non-deterministic Polynomial-hard) problem with multi-variants. Due to various nodes characteristics of cooperative networks, such as, power level of cooperative nodes, corresponding channels fading feature, location-based feature, channel state information (perfect/imperfect), transmission error rate and so on, focusing on this multi- factorial NP-hard problem with high computational complex, therefore, for the sake of realizing optimal transmission subject to these varied restrictive conditions, we need to transform challenging NP-hard/NP-complete problem to P (Polynomial) problem, and propose efficient and ultra-low complexity cooperative fundamental algorithms.

\begin{tabular}{|c|c|c|}
\hline 6G scenarios & Cooperative networks features & Related optimization technology \\
\hline $\begin{array}{l}\text { UAVs communica- } \\
\text { tions }[1,2,7]\end{array}$ & $\begin{array}{l}\text { *Cooperative incentives between segments and cross-layers } \\
\text { protocol design in air-ground networks *Point-to-point com- } \\
\text { munication with CC networks of UAVs *Cooperative air- } \\
\text { terrestrial line-of-sight communications with controlled mo- } \\
\text { bile relays based on Deep reinforcement learning }\end{array}$ & $\begin{array}{l}\text { OFDMA } \\
\text { New networks slicing } \\
\text { Massive MIMO } \\
\text { THz Communications } \\
\text { Artificial intelligence }\end{array}$ \\
\hline $\begin{array}{l}\text { Cognitive IoT net- } \\
\text { works }[1,2,4,6,8]\end{array}$ & $\begin{array}{l}\text { *Cooperative cognitive new spectrum sharing *Multiple ac- } \\
\text { cess *Multiple IOT nodes/sensors CC networks under li- } \\
\text { censed and unlicensed spectrum }\end{array}$ & $\begin{array}{l}\text { Intelligent blockchains } \\
\text { MM-wave communications } \\
\text { Intelligent Reflecting Surfaces }\end{array}$ \\
\hline $\begin{array}{l}\text { Air-space-ground } \\
\text { integrated networks } \\
{[2,3,7]} \\
\text { Free space optical } \\
\text { (FSO)[1,2,7] }\end{array}$ & $\begin{array}{l}\text { *large-scale satellite-aircraft-ground heterogeneous ultra- } \\
\text { mMTC cooperative network *Satellite/plain/ground commu- } \\
\text { nication device-to-device CC networks } \\
\text { *Line-of-Sight (LOS) and short-distance FSO/RF relay CC } \\
\text { networks *Very long-large fronthaul/backhaul Ultra-mMTC } \\
\text { communications }\end{array}$ & $\begin{array}{l}\text { Holographic beamforming } \\
\text { FD-duplex communications } \\
\text { Big data processing and analytics } \\
\text { Device-to-Device optimized communications } \\
\text { Intelligent cloud-fog-edge computation }\end{array}$ \\
\hline $\begin{array}{l}\text { Vehicular networks } \\
{[1,2,5,8]}\end{array}$ & $\begin{array}{l}\text { *Ultra-massive vehicles communications with multiple layers } \\
\text { VoE (Vehicles to Everything) networks architectures *CC } \\
\text { Internet of Vehicles }\end{array}$ & Non-orthogonal multiple access (NOMA, SCMA, PDMA) \\
\hline $\begin{array}{l}\text { Underwater acoustic } \\
\text { communication }[2,6]\end{array}$ & *Underwater cooperative massive sensors networks & \\
\hline
\end{tabular}

TABLE 1. Related optimized techniques in 6G ultra-mMTC hybrid and ubiquitous networks

\section{COOPERATIVE CORE AlgORITHMS IN 6G UlTRA-MMTC NETWORKS}

So far, there are principally two kinds of basic cooperative core algorithms-single node selection and nodes subsets selection, which are extensively linked with related optimization techniques in all sorts of $6 \mathrm{G}$ hybrid scenarios, as other various representative algorithms are also extended theories based on these two types of methods.

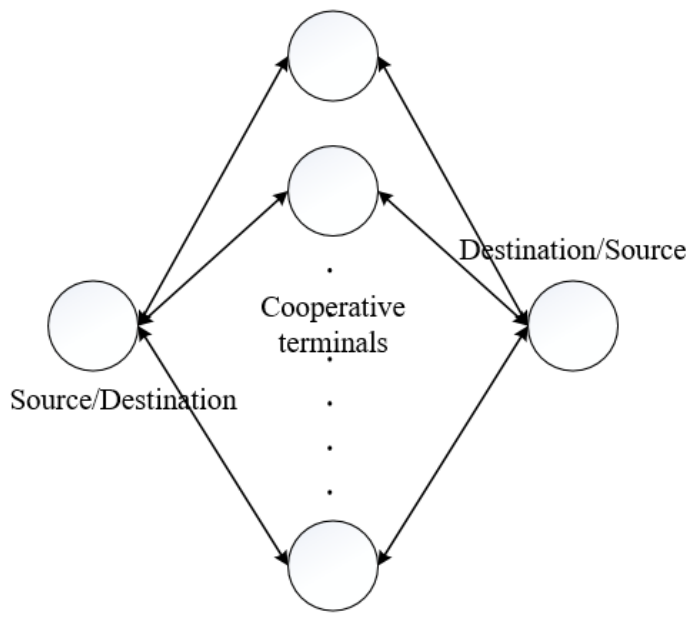

Figure 3. Dual-hop FD cooperative transmission

The former one includes several classical single node selection algorithms: best node selection, $\mathrm{N}$-th best node selection, partial node selection [9, 10, 14]. Here we present these core algorithms in Fig.3 using dual-hop device-to-device FD cooperative communication.

*Best node selection: Selecting node with best SNR (Signal to Noise Ratio) / SINR (Signal to Interference and Noise Ratio) in all cooperative terminals.

*N-th best node selection: Be similar to best selection with eq. (1), selecting node with $\mathrm{N}$-th SNR/SINR in all cooperative terminals.

*Partial node selection: Selecting best SNR/SINR node in partial nodes of all cooperative terminals.

In contrast to single node selection, nodes subsets selection algorithms are put forward to essentially improve communication qualities. Generally speaking, nodes subsets selection are better than single node selection, and best node selection algorithm has better performance than other single node selection algorithms, however, when power of collaborative nodes is fixed, random nodes selection algorithms do not absolutely have superiority over best node selection algorithm, some classical subsets selection with better nodes selection and more efficient power allocation are proposed in [11-15].

*[11] proposes low-complexity and sub-optimal sparse nodes subsets selection algorithms with corresponding beamforming weights under constraints of individual relay power in dual-hop cooperative networks, to maximize SNR at the receiver. Besides $l_{1}$-norm squared and Charnes-Cooper transformation are used for transforming compute complexity of exhaustive search to low optimal-near performance based on the knowledge of second-order statistics of the channels.

*[12] puts forward proactive nodes subsets selection to optimize energy-efficiency of cooperative wireless system, when maximum transmission power is under constraints, so that energy efficiency of system will be maximized with low signaling overhead for larger data packets, comparing with best relay selection and all relays selection. 
*[13] considers minimizing outage probability (OP) at the receiver with semi-distributed amplify-and-forward (AF) relays subsets selection, authors propose relay-ordering-based scheme in the light of a specific metric and source-driven iterative algorithm of power allocation to improve OP with low complexity.

*[14] proposes generalized optimal intelligent cloud-based relays subsets pairing algorithm in B5G/6G cognitive ultralarge-scale/large-scale relays networks, to essentially enhance SINR between source and destination of secondary users, hence channel transmission rate and OP of device-to-device will be greatly improved, in addition, essential principle of future CC (A, B, C theory) has been revealed and proposed generalized algorithm can be converted into almost all sorts of classical algorithms (Best node selection, $\mathrm{N}$-th node selection, partial node selection and various nodes subsets selection), more important, comparing with other relays subsets selection algorithms, raised generalized scheme has extremely lower compute complexity.

*[15] puts forward layers-based optimized mobile relays nodes subsets algorithms for FD-NOMA to solve unsolved millennium issue in B5G/6G Internet of Everything cooperative networks. After comprehensively considering fundamental multiple factors of intelligent relays (satellites, UAVs, vehicles, mobile devices and fixed relays), authors propose a related series of relays subsets theorems to obtain minimum upper bound of maximum device-to-device transmission rates and reveal FD-NOMA two-hop balanced optimal transmission phenomenon in B5G/6G air-space-ground integrated ubiquitous networks. Furthermore, proposed layers-based optimal relays scheme not only can transform synthetic NPhard/NP-complete problem to $\mathrm{P}$ problem with fairly low computation complexity, but also has 1 to $3 \mathrm{~dB}$ advantage than current existing relays subsets selection algorithms.

In general, performance analysis and comparison of core cooperative selection algorithms in future 6G ultra-mMTC networks are listed in Table II. We can observe that multiple nodes subsets schemes are superior to single nodes schemes, and it is more important that proposed CC algorithms should be satisfied with more effective transmission mobile nodes in dynamic networks topology.

\begin{tabular}{|c|c|c|}
\hline Core algorithms & Algorithms description & Rank \\
\hline Nodes subsets selection [15] & $K$-layers based synthesis algorithm (transform NP-hard to $\mathrm{P}$ dynamically) & 1 \\
\hline Nodes subsets selection [14] & *Intelligent cloud-based dual-hop respective subsets pairing & 2 \\
\hline Nodes subsets selection [13] & *Sub-optimal nodes selection and power allocation in a semi-distributed manner & 3 \\
\hline Nodes subsets selection $[12]$ & *Proactive nodes subsets selection to maximum energy-efficiency & 4 \\
\hline Nodes subsets selection [11] & $\begin{array}{l}\text { *Low-complexity and sub-optimal nodes selection with corresponding beam-forming } \\
\text { weights }\end{array}$ & 5 \\
\hline Best node selection [9] & *Best SNR/SINR node selection & 5 \\
\hline$N$-th node selection $[12]$ & *N-th SNR/SINR node selection & 6 \\
\hline Partial node selection $[10]$ & *Best SNR/SINR node selection from partial nodes & 6 \\
\hline
\end{tabular}

TABLE 2. Performance comparison for critical cooperative selection algorithms that will be extensively existing in 6G ultra-mMTC networks

\section{FAST SELECTION ALGORITHM IN ALL SORTS OF 6G UltRA- MMTC NETWORKS}

In order to obtain lower computation complexity, faster selection algorithm of optimal/extreme/sub-optimal massive relays subsets shall be proposed to adapt multiple air-spaceground hybrid communication scenarios. It is noteworthy unlike [10-15], in future 6G all kinds of ultra-mMTC networks we will first comprehensively compute optimal/sub-optimal transmission performance by combining different factors of relays jointly, then implement relays subsets selection. Hence, we creatively considers various different factors of each relay at the same time unusually, and compute all sorts of relays subsets selection to obtain multiple maximum/extreme/suboptimal ultra-low complexity solutions. Noticeably, although it does not provide maximum values combination based on all kinds of relays (fixed base stations, air crafts, satellites, ground mobile devices and so on), it can calculate several different relays combinations to meet different communication scenarios faster than other core cooperative subsets selection schemes in
[9-15].

Shown in Tab.3, we assume there are 80 ultra-massive relays in alternative set which is expressed as $\left\{N_{i} \mid 1 \leq i \leq 80\right\}$, the set has been evenly divided into four alternative classes (each class has 20 relays) as fixed base stations, UAVs, satellites and ground mobile devices, several critical factors have been separately set in actual communication scenarios. We will obtain ultra-massive relays subset selection with constant number. Noticeably, for different relays, such as fixed relays, mobile devices, intelligent cars, UAVs and satellites, because they have their own relays features, for examples, fixed relays will be considered with no mobility and stationary fading feature, line-of-sight communications shall be paid more attention in UAVs communications, relative slow movement shall be taken into account for satellites communications against terrestrial region, thus to different relays, more critical characteristics will be thought over firstly in 6G Internet of Everything air-space-ground integrated networks. 


\begin{tabular}{|c|c|c|c|c|}
\hline & Fixed base station & UAVs & Satellites & Mobile terminals \\
\hline Relay subsets & $\left\{N_{i} \mid 1 \leqslant i \leqslant 20\right\}$ & $\left\{N_{i} \mid 21 \leqslant i \leqslant 40\right\}$ & $\left\{N_{i} \mid 41 \leqslant i \leqslant 60\right\}$ & $\left\{N_{i} \mid 61 \leqslant i \leqslant 80\right\}$ \\
\hline $\begin{array}{l}\text { minimum Channel coef- } \\
\text { ficients }\end{array}$ & $0.6-0.8$ & $0.5-0.7$ & $0.4-0.6$ & $0.55-0.65$ \\
\hline Relay location & Fixed & $\begin{array}{l}\text { Mobile with long distance } \\
\text { LoS communications }\end{array}$ & $\begin{array}{l}\text { Long distance with spatial } \\
\text { fading }\end{array}$ & $\begin{array}{l}\text { Mobile with non-LoS } \\
\text { communications }\end{array}$ \\
\hline $\begin{array}{l}\text { Coefficients of imperfect } \\
\text { channels }\end{array}$ & Mean distribution (0.9-1) & $\begin{array}{l}\text { Mean distribution (0.85- } \\
0.95 \text { ) }\end{array}$ & $\begin{array}{l}\text { Mean distribution (0.8- } \\
0.9)\end{array}$ & $\begin{array}{l}\text { Mean distribution (0.8- } \\
0.9)\end{array}$ \\
\hline $\begin{array}{l}\text { Power allocation for sin- } \\
\text { gle antenna }\end{array}$ & Mean distribution (5-25w) & Mean distribution (5-25w) & Mean distribution (5-25w) & Mean distribution (5-25w) \\
\hline Type of relays & AF/DF/Buffer/Intelligent & AF/DF/Buffer/Intelligent & AF/DF/Buffer/Intelligent & AF/DF/Buffer/Intelligent \\
\hline Network topology & invariant structure & 3D variant structure & $\begin{array}{l}\text { 3D multi-trajectory struc- } \\
\text { ture }\end{array}$ & 2D variant structure \\
\hline
\end{tabular}

Table 3. Parameter setting for 6G ultra-massive relays subsets selection

On the other hand, transmission performance has been substantially improved if more and better relays are preferentially selected, therefore, we will seek solution of multi-team extreme/sub-optimal relays combinations in future multiple 6G scenarios, for example, in town center fixed base stations and ground mobile devices shall be jointly coordinated, but in remote mountain areas satellites and UAVs will be taken into consideration first and selected more, besides, variant network topology and long distance non-LoS/LoS transmission are heavily dependent on fast-moving nodes, and three dimensional mobile relays such as UAVs and air crafts shall firstly be selected in high-altitude communications to construct optimized network transmission structure.

\section{CONCLUSIONS AND CHALLENGES}

This paper points that cooperative ultra-mMTC technology will be fundamental for Internet of Everything in future 6G hybrid and ubiquitous networks, more importantly, ultra-massive collaborative ideology has been regarded as linkage theory for arbitrary two communication nodes, hence, investigating and applying cooperative selection and communication technology is very meaningful for developing $6 \mathrm{G}$ new communication scenarios and substantially improving transmission performance. We firstly establish hybrid 6G air-space-ground Internet of Everything networks topology by integrating various respective 6G networks, secondly we summarize critical techniques that will be applied in future dynamic networks and induce major problems of selection and communication of ultra-massive cooperative relays, next unlike [9-15] that comprehensively consider several or all factors of each relay and implement selection, we discuss novel ultra-massive cooperative relays selection algorithm with fast selection and low computation complexity, and consider selection algorithm shall be used to obtain multiple different relays combinations in all sorts of 6G unique scenarios. In general, development of future Internet of Everything communication are closely linked with collaborative ideology, hence it is very meaningful to establish low complexity and optimal 6G cooperative dynamic topology networks.

\section{ACKNOWLEDGMENT}

This work was supported by National Natural Science Foundation of China (Grant No. XXXXXXXX), China Postdoctoral Science Foundation (Grant No. XXXXXXXX),
National Natural Science Foundations of China under (No.61971092), Sichuan Science and Technology Program (No.2020JDJQ0023), partially supported by Fundamental Research Funds for the Central Universities (No.ZYGX2019J123)

\section{REFERENCES}

[1] P. Yang, Y. Xiao, M. Xiao and S. Li, "6G Wireless Communications: Vision and Potential Techniques," in IEEE Network, vol. 33, no. 4, pp. 70-75, July/August 2019.

[2] M. Z. Chowdhury, M. Shahjalal, S. Ahmed and Y. M. Jang, "6G Wireless Communication Systems: Applications, Requirements, Technologies, Challenges, and Research Directions," in IEEE Open Journal of the Communications Society, vol. 1, pp. 957-975, 2020.

[3] N. Tafintsev et al., "Aerial Access and Backhaul in mmWave B5G Systems: Performance Dynamics and Optimization," in IEEE Communications Magazine, vol. 58, no. 2, pp. 93-99, February 2020.

[4] Q. Cui, W. Ni, S. Li, B. Zhao, R. P. Liu and P. Zhang, "Learning-Assisted Clustered Access of 5G/B5G Networks to Unlicensed Spectrum," in IEEE Wireless Communications, vol. 27, no. 1, pp. 31-37, February 2020.

[5] B. Ji et al., "Survey on the Internet of Vehicles: Network Architectures and Applications," in IEEE Communications Standards Magazine, vol. 4, no. 1, pp. 34-41, March 2020.

[6] I. Tomkos, D. Klonidis, E. Pikasis and S. Theodoridis, "Toward the 6G Network Era: Opportunities and Challenges," in IT Professional, vol. 22, no. 1, pp. 34-38, 1 Jan.-Feb. 2020.

[7] B. Li, Z. Fei and Y. Zhang, "UAV Communications for 5G and Beyond: Recent Advances and Future Trends," in IEEE Internet of Things Journal, vol. 6, no. 2, pp. 2241-2263, April 2019.

[8] X. Shao, X. Chen, D. W. K. Ng, C. Zhong and Z. Zhang, "Cooperative Activity Detection: Sourced and Unsourced Massive Random Access Paradigms," in IEEE Transactions on Signal Processing, vol. 68, pp. 6578-6593, 2020.

[9] Y. Hu, C. Schnelling, M. C. Gursoy and A. Schmeink, "Multi-RelayAssisted Low-Latency High-Reliability Communications With Best Single Relay Selection," in IEEE Transactions on Vehicular Technology, vol. 68, no. 8, pp. 7630-7642, Aug. 2019.

[10] A. Tregancini, E. E. B. Olivo, D. P. M. Osorio, C. H. M. de Lima and H. Alves, "Performance Analysis of Full-Duplex Relay-Aided NOMA Systems Using Partial Relay Selection," in IEEE Transactions on Vehicular Technology, vol. 69, no. 1, pp. 622-635, Jan. 2020.

[11] L. Blanco and M. Nájar, "Sparse Multiple Relay Selection for Network Beamforming With Individual Power Constraints Using Semidefinite Relaxation," in IEEE Transactions on Wireless Communications, vol. 15, no. 2, pp. 1206-1217, Feb. 2016.

[12] B. Klaiqi, X. Chu and J. Zhang, "Energy-Efficient and Low Signaling Overhead Cooperative Relaying With Proactive Relay Subset Selection," in IEEE Trans. Commun., vol. 64, no. 3, pp. 1001-1015, March 2016.

[13] L. Liu, C. Hua, C. Chen and X. Guan, "Semidistributed Relay Selection and Power Allocation for Outage Minimization in Cooperative Relaying Networks," IEEE Trans. Veh. Technol., vol. 66, no. 1, pp. 295-305, Jan. 2017. [14] H.Huang, C.W. Yuan, "Generalized Optimal Two-way Relays Subsets Pairings in Cloud-based Region Cognitive Networks", in IEEE Transaction on Signal Processing, Under Review.

[15] H. Huang, S. Hu, T. Yang and C. W. Yuan, "Full Duplex Non-orthogonal Multiple Access with Layers-based Optimized Mobile Relays Subsets 
Algorithm in B5G/6G Ubiquitous Networks," in IEEE Internet of Things Journal, 2020, pp.1-1.

\section{BIOGRAPHY}

He Huang received his Ph.D degree in school of Information and Communication Engineering, Beijing University of Posts and Telecommunications of China (BUPT), and he is now post-doctor with the National Key Lab on Communications of University of Electronic Science and Technology of China. (UESTC). He has received the Academic Star award in BUPT 2016. He serves as reviewers for several journals, such as, IEEE Transactions on wireless communications, IEEE Transactions on Communications, IEEE Access, IEEE Communication letters, International Journal of Electronics and Physical Communications, and for IEEE Conference WCNC. His main research interests include critical technologies in B5G/6G, cooperative communications, UAV communications, cognitive radios and so on.

$\mathrm{Su} \mathrm{Hu}$ received his M.S. and Ph.D degree in the National Key Lab on Communcations from University of Electronic Science and Technology of China (UESTC) in 2007 and 2010, respectively. Currently, he is now an Professor at the UESTC. From Feb. 2011 to Aug. 2012, He was a Research Fellow in School of Electrical and Electronic Engineering, Nanyang Technological University, Singapore. His research interests include sequence design with good correlation properties and physical layer design for wireless communication systems, such as filterbank multicarrier systems, cognitive radio networks. His homepage is at http://staff.uestc.edu.cn/husu/. 